\title{
MASS-TO-LIGHT RATIO OF INTERMEDIATE AND OLD CLUSTERS IN THE MAGELLANIC CLOUDS
}

\author{
PATRICK SEITZER \\ Space Telescope Science Institute \\ 3700 San Martin Drive \\ Baltimore MC 21218 \\ USA
}

The observed central velocity dispersion of a stellar system is dependent on the mass distribution and the velocity distribution function. For the past several years a program has been undertaken to determine central velocity dispersions of LMC and SMC clusters for comparison with galactic clusters. The mass-to-light ratio (M/L) estimates so obtained should be considerably more accurate than those obtained by tidal arguments (Chun 1978, Elson \& Freeman 1985).

Accurate radial velocities (mean error $=1.3 \mathrm{~km} \mathrm{~s}^{-1}$ ) have been obtained for a sample of K-type giants with the CTIO $4 \mathrm{~m}$ telescope and échelle spectrograph with CCD detector. All of the stars observed were within two core radii of the cluster centre. Multiple observations at several epochs (1984 and 1989) were used to determine the velocity errors.

The central $\mathrm{M} / \mathrm{L}$ in solar units has been estimated from single component isotropic King models (King 1966), with the assumption that the distribution of mass follows that of the light. Observed values for the total integrated light and core radius were taken from the literature (in particular, from Chun 1978, van den Bergh 1981, Mateo 1987).

The results for the three clusters reduced to date are shown in Table 1

Table 1. Mass-to-light ratios for three clusters reduced to date.

\begin{tabular}{llll}
\hline Cluster & Age & $\begin{array}{c}\text { Velocity } \\
\text { dispersion }\end{array}$ & M/L \\
\hline NGC419 & $1 \mathrm{Gyr}$ & $<2 \mathrm{~km} \mathrm{~s}-1$ & $<0.14$ \\
NGC1835 & $>10 \mathrm{Gyr}$ & $6.2 \pm 1.6 \mathrm{~km} \mathrm{~s}-1$ & $0.8 \pm 0.4$ \\
NGC1978 & $2 \mathrm{Gyr}$ & $3.7 \pm 0.8 \mathrm{~km} \mathrm{~s}-1$ & $1.0 \pm 0.4$ \\
\hline
\end{tabular}

No correction has been applied to scale the velocity dispersion to a radius of $r=0$, and so the above values are low by a model dependent factor (typically 10 to $40 \%$ ).

The value at this radius obtained for NGC1835 agrees well with that obtained in integrated light at $\mathrm{r}=0$ by Dubath et al. (1990) for a King model.

For the highly elliptical cluster NGC1978 the value is consistent with that expected for a 2-Gyr old cluster. Note that if the ellipticity of NGC1978 is due to velocity anisotropy, then the true M/L value will be lower. 
But for NGC419 only an upper limit is determined, and that is almost a factor of ten lower than that expected for a cluster of this age (e.g. see Elson et al. 1987). This M/L value is so low that it may be difficult to reconcile it with the observed number and luminosity of stars. One possibility is that I am being misled by the small number of stars with measured velocities (14), since almost any other dynamical effect (binaries, velocity errors, or anisotropy) would cause an overestimate of the $M / L$ ratio. Such a low $M / L$ ratio would be expected for a much younger cluster, but this explanation appears ruled out by the observed colour magnitude diagram.

\section{References}

Chun, M.S. (1978), Astron. J. 83, 1062.

Dubath, P., Meylan, G., Mayor, M., Margain, P. (1990), Astron. Astrophys. in press.

Elson, R.A.W., Freeman, K.C. (1985), Astrophys. J. 288, 521.

Elson, R.A.W., Fall, S.M., Freeman, K.C. (1987), Astrophys. J. 323, 54.

King, I. R. (1966), Astron. J. 71, 64.

Mateo, M. L. (1987), Ph.D. thesis, University of Washington.

van den Bergh, S. (1981), Astron. Astrophys. Suppl. 46, 79. 